\title{
Next-generation sequencing is a powerful method to enhance diagnostic yield in global developmental delay/ intellectual disability
}

Jon Soo Kim, MD, PhD

Department of Pediatrics, Chungbuk National University Hospital, Cheongju, Korea

Next-generation sequencing (NGS) technologies are massively parallel DNA sequencing methods that allow faster and cheaper sequencing of nucleotides than does Sanger sequencing. ${ }^{1)}$ With the advent of NGS, nucleotide sequencing has found applications in the diagnosis of pediatric neurological disorders such as muscle disease, epilepsy, neurodegenerative disease, neurometabolic disease, and autism spectrum disorder. ${ }^{2-4)}$ In recent years, several studies on the genetic diagnosis of unexplained global developmental delay (DD) and intellectual disability (ID) have been published..$^{5-7)}$ Global DD and ID have a high prevalence, affecting $1 \%-3 \%$ of children. ${ }^{8)}$ The diversity of phenotypical and genetical heterogeneity leads to a low diagnostic yield and insufficient clinical management. ${ }^{7)}$

When the clinical picture suggests a syndromic form of DD/ ID, a diagnostic genetic test is used to confirm or rule out chromosomal abnormalities or monogenic disorders: classical

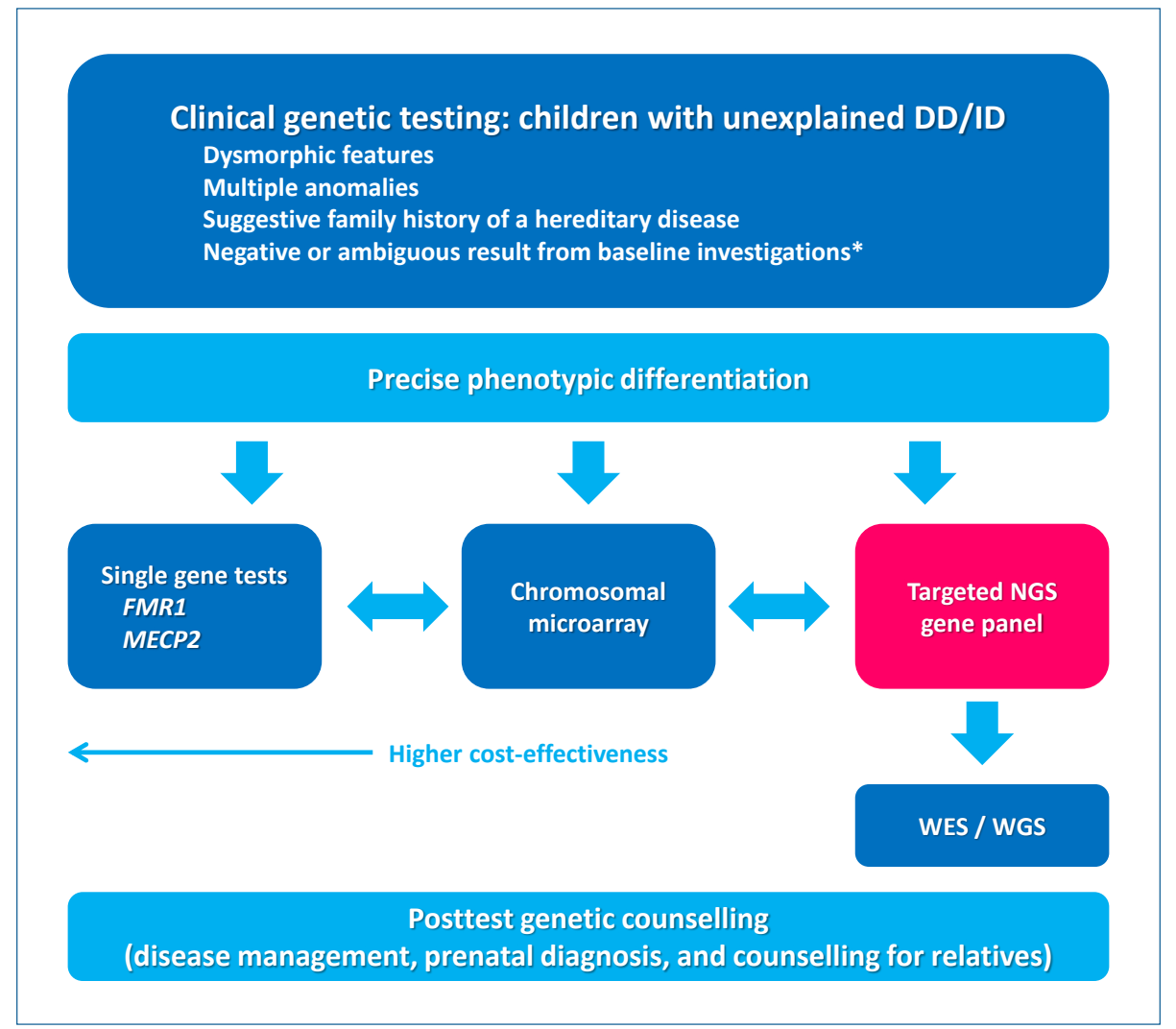

Fig. 1. Current clinical approach to genetic testing in children with developmental delay/intellectual disability. DD, developmental delay; ID, intellectual disability; NGS, next-generation sequencing; WES, wholeexome sequencing; WGS, whole-genome sequencing. *Baseline investigations: biochemistry (blood, urine \pm cerebrospinal fluid, muscle) and brain magnetic resonance imaging.

\footnotetext{
Corresponding author: Jon Soo Kim, MD, PhD. Division of Pediatric Neurology, Department of Pediatrics, Chungbuk National University Hospital, 776 Sunhwan-1-ro, Seowon-gu, Cheongju 28644, Korea 
karyotyping or fluorescence in situ hybridization or molecular biology testing (FMR1 for fragile X syndrome, MECP2 for Rett syndrome). ${ }^{2)}$ As high-resolution chromosomal microarray (CMA) became widely used and provided a much higher diagnostic yield than traditional karyotyping, it became the first-tier of genomic analysis for patients presenting with $\mathrm{DD} / \mathrm{ID}$ in the 2010 American College of Medical Genetics guideline. ${ }^{9)}$ NGS technologies have recently become a key diagnostic tool for establishing a genetic diagnosis in children with DD/ID, reducing the mean cost per diagnosis in cases that are generally more challenging to detect, such as mosaicism. ${ }^{\text {) }}$

Han et al. ${ }^{6}$ ) previously reported a diagnostic yield of $29 \%$ (10 of 25) in Korean patients with DD and/or ID. They suggested that targeted NGS through singleton analysis with a phenotypefirst approach is useful for genetic diagnosis. In this review article, Han emphasized a broad acceptance of NGS applications such as targeted panel sequencing (TPS), whole-exome sequencing (WES), and whole-genome sequencing (WGS) with reduced cost. Suggested guidelines for genetic investigations in children with DD/ID recommend TPS as the first-tier test and familybased trio testing by WES/WGS as the second-tier test, although a few technical limitations such as detecting microdeletions or duplications and mutations in noncoding trinucleotide expansion diseases. ${ }^{10)}$ Accordingly, an updated clinical approach to genetic investigations in children with DD/ID is shown in Fig. 1. ${ }^{9,11)}$ The first step in increasing the diagnostic yield is to clearly define the population of suspected genetic causes that have dysmorphic features, multiple anomalies, suggestive family history of a hereditary disease, and negative or ambiguous results from baseline investigations. Another important consideration is that more detailed and precise phenotypic differentiations lead to a higher diagnostic yield of genetic diagnosis. After differentiating phenotypes, clinicians can choose the first-tier test with consideration of cost-effectiveness: single gene tests, CMA, or TPS. WES/WGS can help to identify a causative genomic variant in cases in which previous testing was inconclusive. Once a genetic diagnosis is established, posttest genetic counseling should address possible therapeutic interventions and the risk of recurrence.
There are still many considerations related to advanced genetic testing that continuously reclassifying variants of unknown significance and ethical issues. ${ }^{11)}$ Nonetheless, the role of NGS testing in the clinical setting is becoming more important and clinicians should be aware of its rapid changes in this genetic era.

See the article "Genetic tests by next-generation sequencing in children with developmental delay and/or intellectual disability" via https://doi.org/10.3345/kjp.2019.00808.

\section{References}

1. Park ST, Kim J. Trends in next-generation sequencing and a new era for whole genome sequencing. Int Neurourol J 2016;20:S76-83.

2. Valente EM, Ferraris A, Dallapiccola B. Genetic testing for paediatric neurological disorders. Lancet Neurol 2008;7:1113-26.

3. Ko A, Kang HC. Frequently identified genetic developmental and epileptic encephalopathy: a review focusing on precision medicine. Ann Child Neurol 2019;27:2-12.

4. Marques Matos C, Alonso I, Leão M. Diagnostic yield of next-generation sequencing applied to neurological disorders. J Clin Neurosci 2019; 67:14-8.

5. Li Y, Anderson LA, Ginns EI, Devlin JJ. Cost effectiveness of karyotyping, chromosomal microarray analysis, and targeted next-generation sequencing of patients with unexplained global developmental delay or intellectual disability. Mol Diagn Ther 2018;22:129-38.

6. Han JY, Jand JH, Park J, Lee IG. Targeted next-generation sequencing of Korean patients with developmental delay and/or intellectual disability. Front Pediatr 2018;6:391.

7. Harripaul R, Noor A, Ayub M, Vincent JB. The use of next-generation sequencing for research and diagnostics for intellectual disability. Cold Spring Harb Perspect Med 2017;7(3). pii: a026864. https://doi.org/ 10.1101/cshperspect.a026864.

8. Maulik PK, Mascarenhas MN,MathersCD, Dua T, Saxena S. Prevalence of intellectual disability: a meta-analysis of population-based studies. Res Dev Disabil 2011;32:419-36.

9. Srour M, Shevell M. Genetics and the investigation of developmental delay/intellectual disability. Arch Dis Child 2014;99:386-9.

10. Han JY, Lee IG. Genetic tests by next-generation sequencing in children with developmental delay and/or intellectual disability. Clin Exp Pediatr 2020 Feb 6. [Epub]. https://doi.org/10.3345/kjp.2019.00808.

11. Vasudevan P, Suri M2. A clinical approach to developmental delay and intellectual disability. Clin Med (Lond) 2017;17:558-61. 